\section{Un trio de mécanismes au cour de l'initiation de la régénération chez les animaux}

Aurore Vullien ${ }^{1,2}$, Éric Röttinger ${ }^{2,3}$, Michel Vervoort ${ }^{1}$, Eve Gazave ${ }^{1}$

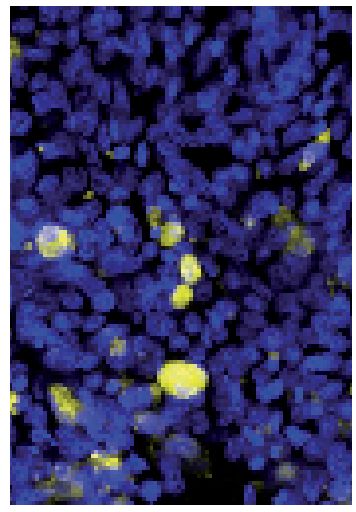

${ }^{1}$ Université de Paris, CNRS, Institut Jacques Monod, F-75006 Paris, France. 2Université Côte d'Azur, CNRS, Inserm, IRCAN (Institute for Research on Cancer and Aging), 28 avenue de Valombrose, Nice, France. ${ }^{3}$ Université Côte d'Azur, Institut fédératif de recherche Ressources marines, Nice, France. eve.gazave@ijm.fr

\section{Importance et diversité de la régénération chez les animaux}

La régénération, c'est-à-dire la reformation d'une partie du corps perdue, est un processus fascinant qui intéresse les scientifiques et les naturalistes depuis des siècles. Deux grands types de régénération peuvent être distingués $[1,2]$ : une régénération apparaissant dans un contexte homéostatique, tel que le remplacement de l'épiderme et de ses dérivés (poils, cheveux, etc.) ou de l'épithélium digestif, et celle survenant à la suite d'une blessure ou d'une amputation. Cette dernière, qui est le sujet de cette revue, est très largement répandue chez les animaux, même si les capacités régénératives varient grandement d'une espèce à l'autre (Figure l) [2]. Certains animaux, comme les vers plats (ou plathelminthes), sont capables de régénérer un individu entier à partir d'un petit fragment corporel. D'autres, comme certains arthropodes et certains vertébrés, peuvent reformer des structures complexes, telles que des pattes ou des nageoires. D'autres encore ont des capacités plus limitées et ne peuvent régénérer que cer-

Vignette (Photo @ Eve Gazave) tains organes (le foie chez les mammifères, par exemple) ou certains tissus (notamment les muscles). Enfin, certains animaux, tels que les nématodes, ne semblent être capables de régénérer que certains de leurs axones. Pourquoi les capacités de régénération sont-elles très limitées chez certaines espèces et tellement importantes chez d'autres reste une question largement ouverte.

Outre son intérêt pour la recherche fondamentale, la régénération intéresse également un nouveau pan de la recherche médicale, la médecine régénérative. Alors que les chercheurs de ce domaine se sont longtemps focalisés sur le potentiel des cellules souches, nombre d'entre eux s'intéressent désormais à la possibilité de stimuler la régénération à partir de tissus déjà en place. II est donc devenu nécessaire de déchiffrer les mécanismes qui permettront à la régénération d'être complète et réussie [3]. Dans cette Synthèse, nous décrivons et discutons les mécanismes responsables de l'initiation de la régénération. Nous nous intéresserons plus particulièrement aux rôles des dérivés réactifs de l'oxygène (DRO) (en anglais, ROS pour reactive oxygen species), de l'apoptose, et de la prolifération cellulaire. Nous présenterons également les voies de signalisation qui permettent le lien entre ces trois mécanismes clés.

\section{Prolifération cellulaire et formation d'un blastème de régénération}

Malgré la diversité des modalités de régénération, il est possible de subdiviser ce processus en trois grandes étapes, qui sont retrouvées chez la plupart des animaux ayant des capacités régénératives [4]. 


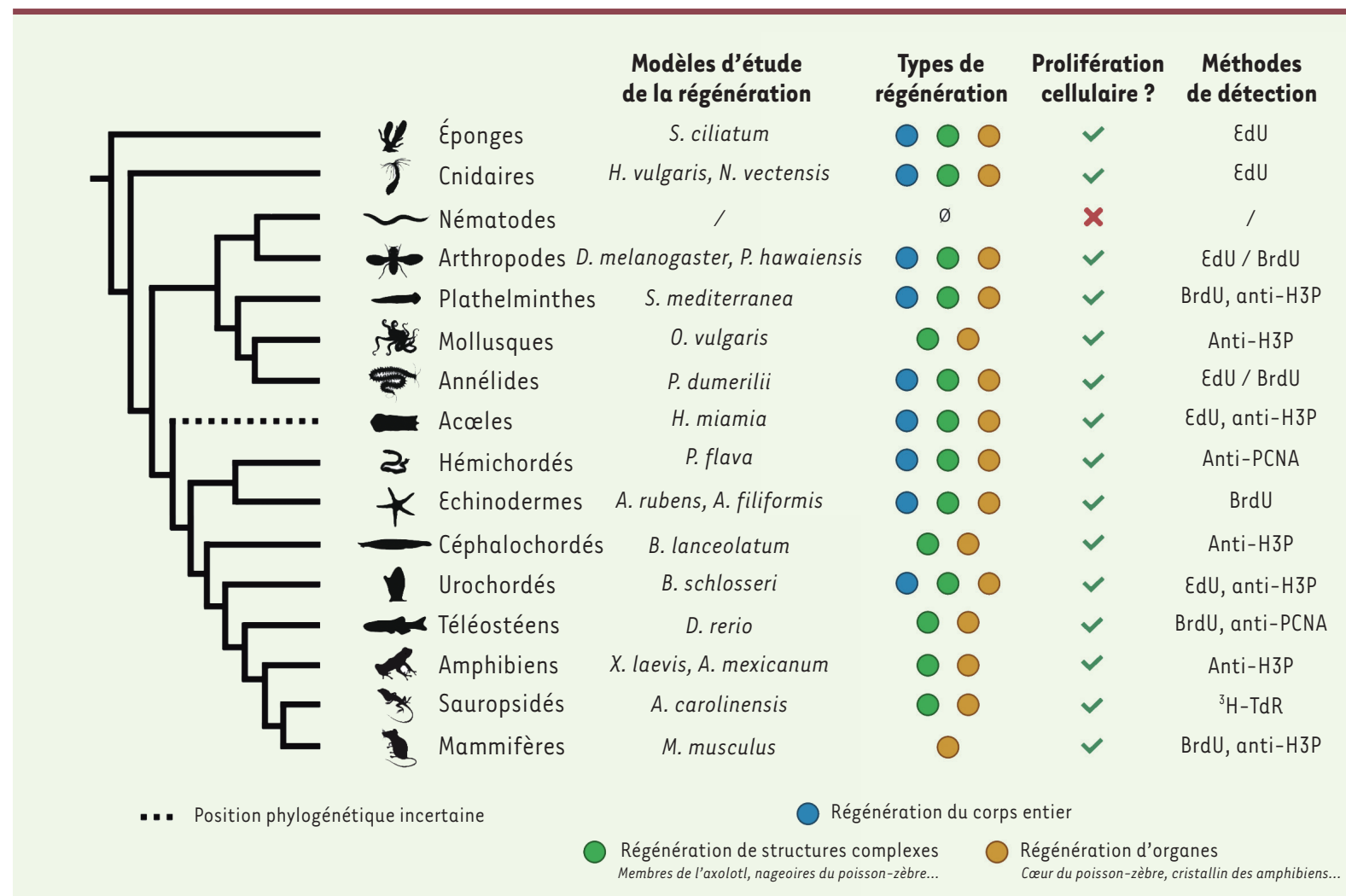

Figure 1. Capacités régénératives et prolifération cellulaire au cours de la régénération chez les animaux. L'arbre phylogénétique simplifié (à gauche) montre quelques groupes d'animaux. Pour chaque groupe, sont indiqués (de gauche à droite) le nom d'une ou deux espèces modèles principales chez lesquelles la régénération est étudiée, quelles sont les capacités régénératives retrouvées, si un épisode d’hyper-prolifération cellulaire a été observé au cours de la régénération, et quelles techniques ont été employées pour mettre en évidence cette hyper-prolifération. Pastille bleue : capacité à régénérer le corps entier. Pastille verte : capacité à régénérer des structures complexes, comme les membres chez l'axolotl ou les nageoires chez le poisson-zèbre. Pastille jaune : capacité à régénérer des organes, comme le cœur du poisson-zèbre ou le cristallin de certains amphibiens. Le 5-éthynyl-2'-désoxyuridine ( $(\mathrm{dU} U)$, le 5-bromo-2'-désoxyuridine (BrdU) et la thymidine tritiée ( ${ }^{3} \mathrm{H}$ - $\left.\mathrm{TdR}\right)$ sont des analogues de nucléotides qui permettent de détecter les cellules en train de proliférer. Les anticorps dirigés contre les protéines PCNA (proliferating cell nuclear antigen) (anti-PCNA) et contre la forme phosphorylée de l'histone H3 (anti-H3P) permettent également de mettre en évidence la prolifération des cellules. Les noms des espèces modèles sont indiqués sous forme abrégée. Les noms complets sont (de haut en bas) : Sycon ciliatum, Hydra vulgaris, Nematostella vectensis, Drosophila melanogaster, Parhyale hawaiensis, Schmidtea mediterranea, Octopus vulgaris, Platynereis dumerilii, Hofstenia miamia, Ptychodera flava, Asterias rubens, Amphiura filiformis, Branchiostoma lanceolatum, Botryllus schlosseri, Danio rerio, Xenopus laevis, Ambystoma mexicanum, Anolis carolinensis, Mus musculus (modifié d'après [22, 36] et réalisé à l'aide de BioRender.com).

Après une blessure ou une amputation, survient rapidement une première étape, dite de cicatrisation, à l'issue de laquelle la blessure est recouverte par un épithélium de cicatrisation qui restaure l'intégrité du milieu intérieur de l'animal. S'ensuit une seconde étape, constituée par la mise en place et l'activation des cellules précurseurs à l'origine des futures structures régénérées. Chez certaines espèces, cette étape repose sur la formation d'une structure spécifique, appelée blastème de régénération, qui consiste en une masse de cellules indifférenciées recouverte d'un épithélium, et à partir de laquelle seront reformées les parties perdues [1] au cours d'une troisième et dernière étape appelée morphogenèse. Au cours de cette étape, les différentes structures sont régénérées et leurs cellules, tissus et organes constitutifs se différencient et retrouvent leurs rôles initiaux.
La prolifération cellulaire joue généralement un rôle important au cours des différentes étapes de la régénération. Les cellules du blastème se divisent activement, produisant ainsi les très nombreuses cellules nécessaires à la formation des parties du corps qui seront régénérées. La formation du blastème lui-même dépend de la prolifération de cellules précurseurs, ce qui survient quelques dizaines d'heures ou quelques jours après l'amputation et la cicatrisation. Cette hyper-prolifération consécutive à l'amputation a pu être observée, notamment grâce à l'utilisation d'analogues modifiés de nucléotides, qui permet de visualiser et de quantifier les cellules en phase de synthèse d'ADN (phase $S$ du cycle 
cellulaire) à différents moments après l'amputation (Figure 1) [5-7]. Chez divers animaux très distants d'un point de vue évolutif, l'application, après une amputation, de molécules qui bloquent les divisions cellulaires empêche la régénération des structures amputées, ce qui montre l'importance des divisions cellulaires pour la réussite de cette régénération [6-8]. Dès lors, la question qui se pose est d'identifier les mécanismes et les signaux qui stimulent cette prolifération lors de l'initiation de la régénération. Une première réponse, inattendue, à cette question a été trouvée : la mort cellulaire par apoptose.

\section{L’apoptose, un acteur clé de la régénération}

L'apoptose est une mort cellulaire programmée : les cellules concernées orchestrent elles-mêmes leur destruction en réaction à des signaux internes ou externes (Figure 2A). Les acteurs emblématiques de l'apoptose sont les caspases, des protéases capables de couper d'autres protéines et ainsi d'en modifier l'activité. Ce processus est à l'origine de la mort de la cellule, notamment après une fragmentation de son ADN [9]. Les signaux conduisant à l'activation des caspases dites initiatrices peuvent être externes ou intracellulaires, définissant ainsi les deux grandes voies de l'apoptose : la voie extrinsèque, activée par la fixation de molécules appelées ligands de mort, produites par d'autres cellules, sur des récepteurs exprimés à la membrane des cellules destinées à mourir ; et la voie intrinsèque, qui se déclenche en réponse à un stress intracellulaire, par exemple, des altérations importantes de l'ADN [43] (Figure 2A) $(\rightarrow)$. $(\rightarrow)$ Voir le numéro et $\mathrm{mort}, \mathrm{m} / \mathrm{s} \mathrm{n}^{\circ} 12$, décembre 2020 thématique Vieillissement

Deux des rôles de l'apoptose sont bien connus: l'élimination des cellules dysfonctionnelles, notamment celles présentant de trop nombreuses coupures de leur ADN ; l'élimination des cellules surnuméraires produites lors du développement de certains tissus, contribuant ainsi au façonnement et à la fonctionnalité de certains organes. La mise en place du cerveau et des doigts des mammifères sont des exemples représentatifs de l'importance de l'apoptose au cours du développement [10].

Un rôle clé de l'apoptose a été mis en évidence au cours de la régénération [11]. Des épisodes d'apoptose ont en effet été observés après une amputation chez différents organismes modèles (Figure 3), grâce à différents types de marquages moléculaires, notamment en utilisant la méthode TUNEL (terminal deoxynucleotidyl transferase dUTP nick end labeling). Celle-ci repose sur l'incorporation d'analogues de nucléotides aux extrémités libres de l'ADN, révélant ainsi les cassures du génome caractéristiques des cellules mourantes. Elle est fréquemment employée en association avec d'autres marquages, notamment ceux effectués avec des anticorps dirigés contre la forme active des caspases [12]. Plus récemment, des senseurs génétiques, permettant de suivre l'activité des caspases in vivo dans des tissus vivants, ont été développés $[13,14]$. II s'agit de protéines fluorescentes produites dans des organismes génétiquement modifiés et dont la fluorescence ou la localisation subcellulaire sont modifiée par l'activité des caspases (Figure 2B).
Chez l'hydre d'eau douce (Hydra vulgaris), une unique vague d'apoptose a lieu dans les deux heures suivant l'amputation (ou heures post-amputation, hpa) [15]. Au cours de la régénération du corps entier chez l'anémone de mer Nematostella vectensis [16], de même que lors de la régénération de la nageoire caudale chez le poissonzèbre Danio rerio [17], deux pics de mort cellulaire sont détectés. Chez le poisson-zèbre, le premier pic (entre 0 et $10 \mathrm{hpa}$ ) pourrait correspondre à la nécrose induite par la blessure. Le second pic, qui apparaît vers 15 hpa, correspond à une apoptose. Chez la drosophile (Drosophila melanogaster), l'induction d'une blessure des disques imaginaux (des structures présentes chez la larve à l'origine de différents organes chez l'adulte, comme les ailes et les yeux) est suivie de la mort des cellules au site de la blessure, mais aussi de celle de cellules de territoires adjacents [18].

Des expériences d'inhibition pharmacologique réalisées au moyen de molécules telles que le Z-VAD ${ }^{1}$, un inhibiteur universel de caspases, ou le NS36942, un inhibiteur de l'apoptosome ${ }^{3}$ (Figure 2A), suggèrent que l'apoptose joue un rôle important dans le bon déroulement de la régénération. Le traitement par l'un ou l'autre de ces inhibiteurs empêche en effet la formation du blastème et une régénération normale, chez l'hydre [15], le poisson-zèbre [17] et l'anémone de mer [16]. La prolifération cellulaire, essentielle à la régénération, n'a pas lieu chez les organismes traités avec ces inhibiteurs de l'apoptose. Chez la larve de drosophile, des expériences de génétique moléculaire ont permis d'induire l'entrée en apoptose de cellules des disques imaginaux tout en empêchant leur mort effective et leur élimination [19]. La persistance de ces cellules «mortes-vivantes» s'accompagne d'une hyper-prolifération des cellules voisines. L'apoptose de certaines cellules pourrait donc stimuler la prolifération de cellules avoisinantes au cours de la régénération, comme le suggère d'ailleurs l'organisation temporelle des pics de mort et de prolifération cellulaire, qui se suivent ou se chevauchent chez ces espèces [11]. Le terme «prolifération compensatoire induite par l'apoptose » (en anglais apoptosisinduced compensatory proliferation) [20] s'est imposé pour désigner ce phénomène. L'apoptose jouerait aussi d'autres rôles au cours de la régénération, lors de la migration et de la différenciation cellulaire, ainsi qu'au cours de la réponse immunitaire induite par l'amputation [21].

\footnotetext{
${ }^{1} \mathrm{~N}$-Benzyloxycarbonyl-Val-Ala-Asp (0-Me).

${ }^{2}$ 4-chloro-2-[3-(3-trifluoromethyl-phenyl)-ureido]benzoic acid.

${ }^{3}$ Complexe multimoléculaire formé par l'interaction du cytochrome avec l'Apaf-1 (apoptosis activating factor 1 ) et la procaspase-9. II permet le clivage de la pro-
} caspase 9 , ce qui induit la formation de la forme active de cette enzyme. 

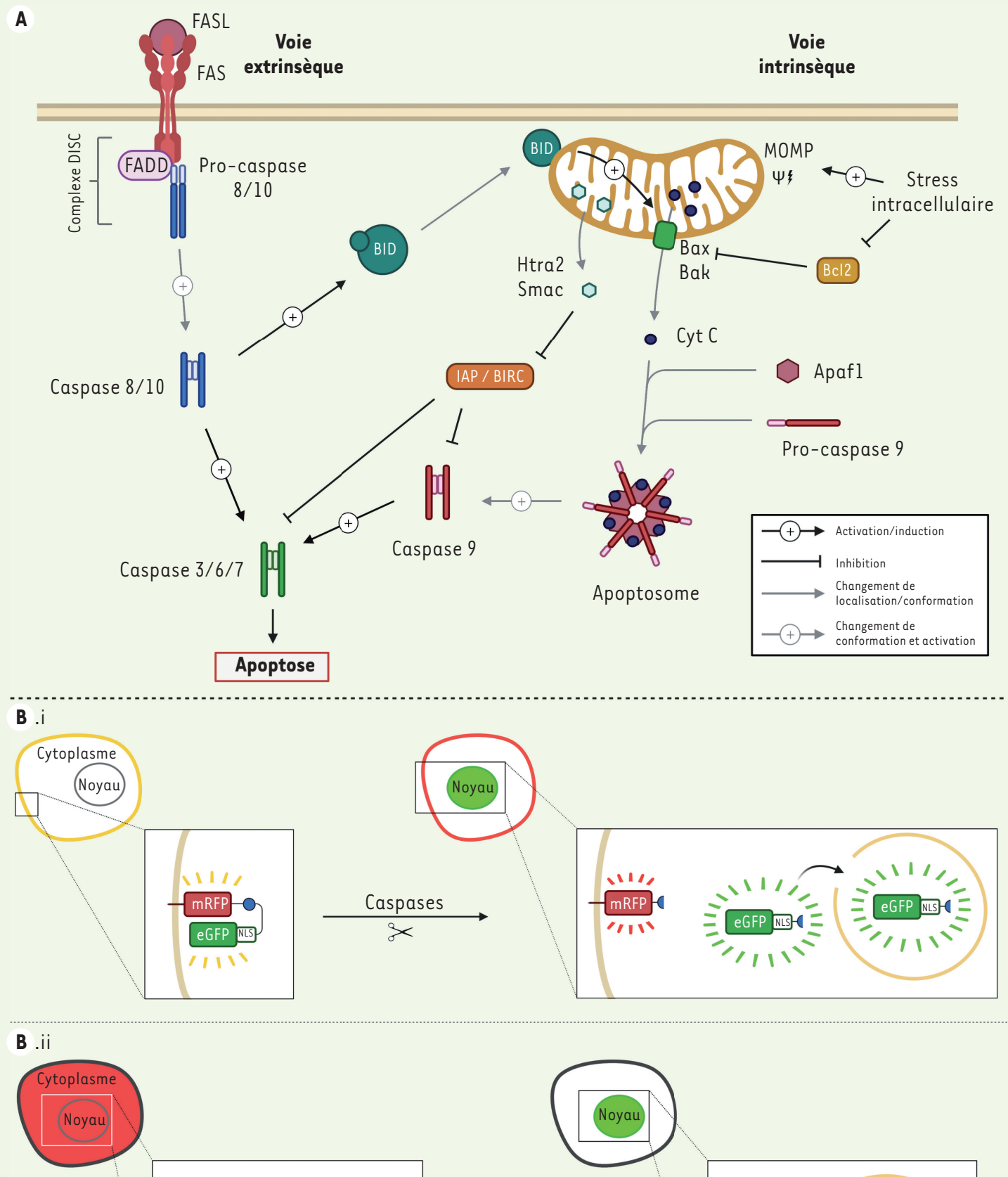

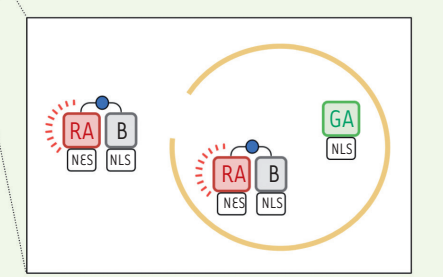

\section{Quels sont les signaux émis par les cellules en apoptose qui stimulent la prolifération cellulaire?}

Chez plusieurs espèces animales, l'induction du processus d'apoptose joue donc un rôle essentiel dans l'initiation de la prolifération cellulaire requise pour une régénération réussie. Les cellules en apoptose induisent en effet, via l'émission de signaux mitogènes, l'entrée en division des cellules avoisinantes (Figure 3). L'identité de ces molécules mitogènes n'est cependant actuellement connue que dans un nombre très réduit de cas de régénération. Elles pourraient différer (au moins partiellement) selon les espèces et les types 
Figure 2. Voies d'induction de l'apoptose chez les mammifères et senseurs génétiques permettant son étude. (A) Les acteurs emblématiques de l'apoptose sont les caspases, dont on distingue deux types : les caspases initiatrices ( 8 , 9 , et 10 chez les mammifères), dotées d'un domaine spécifique permettant leur recrutement dans des complexes protéiques (voir ci-dessous), au sein desquels elles passent d'un état inactif (pro-caspase) à un état actif (caspase) en se coupant elles-mêmes (auto-clivage) ; les caspases effectrices ( $3,6,7$ chez les mammifères), activées après leur coupure par les caspases initiatrices et induisant la mort de la cellule, en clivant elles-mêmes de nombreuses protéines ( $\alpha$-tubuline, DNases, etc.) [37]. La voie extrinsèque implique la fixation de ligands extracellulaires (comme FAS ligand: dérivé de FS-7-associated surface antigen) à des récepteurs de mort membranaires (comme FAS), provoquant le recrutement d'un complexe protéique appelé DISC (death-inducing signaling complex) constitué de FADD (FAS-associated proteins with death domains) et des caspases initiatrices sous leur forme inactive (pro-caspases). Ce recrutement permet l'activation des caspases $8 / 10$, ce qui conduit à l'activation des caspases effectrices 3/6/7. Dans le cas de la voie extrinsèque, des signaux de stress intracellulaires conduisent à la perméabilisation de la membrane mitochondriale (MOMP : mitochondrial outer membrane permeabilization). Cette perméabilisation est gouvernée par l'action antagoniste de protéines de la famille Bcl2, pro-apoptotiques (Bax, Bak, Bid, Bok) et anti-apoptotiques (Bcl2, BCL2L1/2/3, BCL2A1). Plusieurs facteurs sont alors libérés dans le cytoplasme. Htra2 et Smac sont des inhibiteurs des protéines IAP/BIRC (inhibitors of apoptosis, aussi appelés baculoviral IAP repeat-containing protein), elles-mêmes inhibitrices de différentes caspases. Le cytochrome $C$, libéré via des canaux formés par Bax et Bak, s'associe à Apafl et à la pro-caspase 9 pour former l'apoptosome. Ce complexe permet le clivage de cette dernière en caspase 9, qui, à son tour, active les caspases 3, 6 et 7 . Les deux voies ne sont pas indépendantes: ainsi les caspases 8/10, impliquées dans la voie extrinsèque, peuvent activer, en la clivant, la protéine pro-apoptotique BID, qui favorise la perméabilisation mitochondriale (schéma modifié d'après [38]). (B) Les senseurs génétiques, Apoliner (B.i) et FPX (échange de protéine fluorescente ou fluorescent protein exchange), (B.ii) permettent de mettre en évidence l'activité des caspases au sein des tissus vivants. (B.i) Le senseur Apoliner a été mis au point chez des embryons de drosophile et de poulet par Bardet et al. en 2008 [13]. Ce senseur comprend deux protéines fluorescentes différentes : mRFP (fluorescence rouge, protéine membranaire) et eGFP (fluorescence verte, protéine nucléaire du fait de la présence dans sa séquence d'un domaine de localisation nucléaire, NLS pour nuclear localisation signal), reliés par une séquence (disque bleu) reconnue et clivée par les caspases effectrices (séquence appelée CCS pour caspase cleavage sequence). En absence d'activité des caspases, le senseur est localisé à la membrane plasmique et y induit une fluorescence à la fois rouge et verte. La membrane apparait donc jaune. En cas d'activité des caspases, après le clivage de la CCS, les deux protéines fluorescentes se séparent l'une de l'autre. eGFP est transportée dans le noyau, y produisant une fluorescence verte, tandis que mRFP reste associée à la membrane, où l'on observe une fluorescence rouge. (B.ii) Le système FPX a été développé par Ding et al. en 2015 [14], en utilisant des cellules de mammifères en culture. II est fondé sur l'emploi de protéines qui ne sont initialement pas fluorescentes (protéines GA et RA), mais qui le deviennent en s'associant à une autre protéine (protéine B). Le système repose sur la production dans les cellules de B et de RA qui sont liées l'une à l'autre par une séquence CCS (disque bleu), et de GA. Cette dernière, seule, n'est pas fluorescente, alors que RA, qui est associée à B, émet de la fluorescence rouge. Des séquences de localisation nucléaire NLS ou extranucléaire (NES, pour nuclear export sequence) positionnent la molécule non-fluorescente GA dans le noyau, tandis que le complexe RA-B fluorescent, rouge, est présent dans toute la cellule. C'est la situation qui est observée en absence d'activité des caspases. En présence de caspases actives, après le clivage de la séquence CCS, B va se dissocier de RA (qui ne va donc plus émettre de fluorescence) et s'associe à $G A$, présent dans le noyau. Le complexe GA-B émet alors de la fluorescence verte et le noyau devient donc vert.

de régénération. Nous ne détaillerons ici que quelques-unes de ces molécules (pour une liste détaillée de ces molécules et des voies de signalisation qui leur sont associées, voir [11, 22]).

Une étude pionnière, portant sur la régénération de la tête chez l'hydre, a montré que les cellules apoptotiques émettaient des protéines $W_{n t} 3^{4}$, et que celles-ci étaient nécessaires à la prolifération cellulaire et à une régénération correcte [15]. Wnt3 appartient à la grande famille des protéines de signalisation Wnt, connues pour leurs multiples rôles au cours du développement, de la régénération et de la cancérogenèse [23]. Notons néanmoins que la production de protéines Wnt par des cellules apoptotiques n'a été clairement montrée, à ce jour, que dans un seul autre cas: la régénération homéostatique de l'épiderme chez le poisson-zèbre [24]. Lors de ce processus, de petites vésicules extracellulaires (appelées corps apoptotiques) émises à la suite de l'apoptose des cellules et contenant des protéines Wnt8a,

${ }^{4}$ Des protéines de la famille Wnt dérivant des premiers membres identifiés chez la drosophile (Wingless) et chez la souris (Int-1), impliqués dans de nombreux processus lors du développement. induisent la division de cellules voisines, en fusionnant avec ces cellules, via l'activation de la voie de signalisation Wnt/ $\beta$-caténine. La question de l'existence de tels mécanismes impliquant des protéines Wnt dans d'autres cas de régénération reste discutée.

Une autre observation récente a montré que des cellules apoptotiques pouvaient influencer des cellules avoisinantes via la sécrétion ciblée de certains métabolites, notamment des nucléotides ou des molécules produites lors de la glycolyse ou du métabolisme des acides aminés [25]. Ces molécules, dont la sécrétion dépend de l'activité des caspases, induisent de multiples mécanismes dans les cellules voisines, notamment leur prolifération. Notons que ces données ont été obtenues dans un contexte de cultures de cellules in vitro. L'existence ainsi que le rôle de telles sécrétions dans un contexte de régénération in vivo restent donc à démontrer et à étudier. Ces données font néanmoins écho au rôle de 


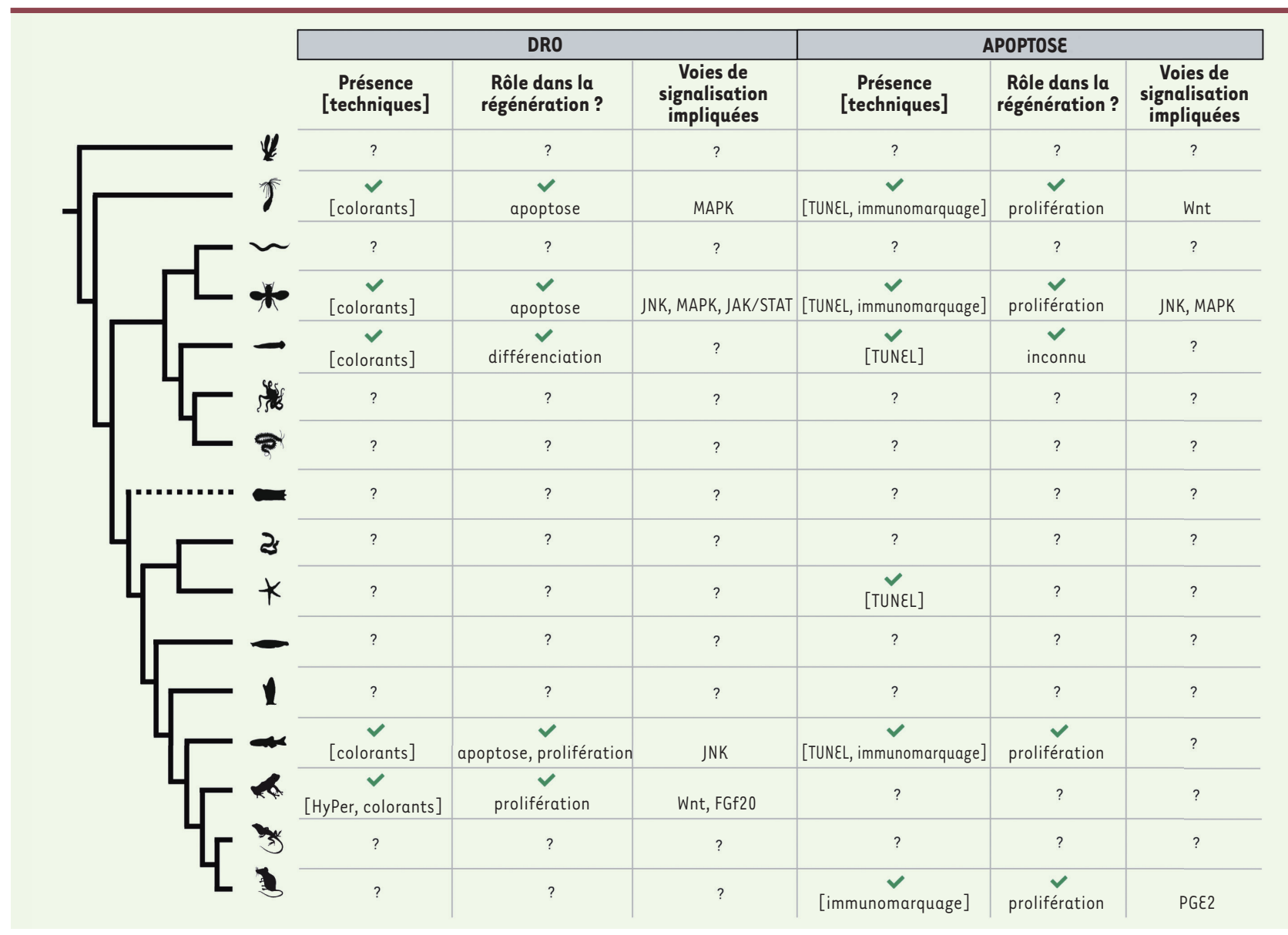

Figure 3. Synthèse des données connues sur l'implication de l'apoptose et des DRO au cours de la régénération. L'arbre phylogénétique de la Figure 1 est repris. Pour chaque groupe, il est indiqué si la présence d'apoptose ou de DRO a été mise en évidence au cours de la régénération chez au moins une espèce de ce groupe, quelle(s) technique(s) a (ont) permis de détecter cette présence, si des études fonctionnelles ont établi que ces mécanismes sont nécessaires à la poursuite de la régénération, si un rôle dans le contrôle de l'apoptose et de la prolifération a été mis en évidence, et, enfin, quelles sont les voies de signalisation impliquées. En fonction des techniques utilisées, la coloration indique que la mise en évidence des DRO s'est faite grâce à des colorants sensibles à la présence de DRO dans les cellules. HyPer est un senseur génétique de $\mathrm{H}_{2} \mathrm{O}_{2}$ et est décrit dans la Figure 4. La méthode TUNEL est décrite dans le texte principal, et «immunomarquage » indique l'utilisation d'anticorps marquant les cellules en apoptose, en particulier celle d'anticorps dirigés contre la forme clivée active des capsases effectrices. L'ensemble des informations de cette figure a été synthétisé à partir de [11,22].

I'ATP (et de ses métabolites, comme l'adénosine) dans la stimulation de la prolifération au cours de la régénération chez certains animaux [26].

Les cellules apoptotiques semblent donc capables de produire divers signaux induisant la prolifération des cellules voisines. Mais quels sont les signaux qui causent l'entrée en apoptose de certaines cellules? Plusieurs études indiquent un signal commun, la production de DRO, faisant suite à la blessure ou à l'amputation.

\section{Rôle des dérivés réactifs de l'oxygène dans l'initiation de la régénération}

Un dérivé réactif de l'oxygène est une molécule oxygénée qui présente un électron libre à l'origine de sa forte réactivité chimique. Le plus connu est sans doute le peroxyde d'hydrogène $\left(\mathrm{H}_{2} \mathrm{O}_{2}\right)$. Les DRO sont produits par la chaîne respiratoire mitochondriale ou par certains complexes enzymatiques (Figure 4A). Ils peuvent être inhibés par le système antioxydant de la cellule, constitué de différentes enzymes qui les catabolisent en eau et dioxygène. Ils peuvent également être à l'origine de radicaux hydroxyles qui seront responsables du stress oxydant, une agression des composants cellulaires par les radicaux libres produits. Ces derniers sont en effet capables d'altérer les macromolécules de la cellule et ne sont pas éliminés par des réactions enzymatiques.

Si les DRO ont longtemps été considérés uniquement comme une cause de stress oxydant dans les cellules, 
ils sont désormais également reconnus comme des acteurs majeurs de la signalisation intercellulaire [27]. Ils ont un rôle important au cours de la régénération. En effet, après des amputations ou des blessures, une production de DRO a été observée à proximité des lésions chez différentes espèces, comme le xénope [28], la drosophile [29], le poisson-zèbre [17] et l'hydre [11] (Figure 3). Ces observations ont été faites au moyen de molécules comme le dihydroéthidium (DHE), dont la fluorescence est sensible à la présence de DRO $\mathrm{H}_{2} \mathrm{O}_{2}$, acteur majeur de la signalisation par les DRO, peut également être révélé dans les tissus vivants grâce à des molécules fluorescentes sensibles à sa présence $[30,31]$, produites chez des animaux génétiquement modifiés (Figure 4B).

La production de DRO, consécutive à une amputation, s'est révélée indispensable au bon déroulement de la régénération chez différentes espèces. Après l'amputation de la queue du têtard de xénope ${ }^{5}$ [28] ou de celle de l'axolot $\left.\right|^{6}$ [32], la blessure des disques imaginaux de larves de drosophile [29], ou l'amputation de la nageoire caudale du poisson-zèbre [17], l'inhibition de la production de DRO empêche en effet la régénération de la structure lésée.

Chez certaines espèces modèles, les pics d'apoptose et les vagues de prolifération cellulaire observés au cours de la régénération n’ont pas lieu en l'absence de production de DRO, indiquant que cette production est nécessaire à l'induction de l'apoptose. Chez la drosophile, la surexpression d'enzymes métabolisant les DRO, ou l'inhibition de leur production, affecte le processus de régénération. L'épisode d'apoptose, ainsi que la prolifération cellulaire qu'il induit, requis pour la régénération, n'ont en effet pas lieu dans ces conditions [29]. Chez le poisson-zèbre, l'inhibition de l'activité de l'enzyme responsable de la production de DRO est à l'origine d'une diminution du nombre de cellules apoptotiques et d'une réduction de la taille du blastème de régénération, empêchant, de ce fait, la régénération correcte de la structure amputée [17].

Les voies de signalisation mises en œuvre dans ces processus ne sont encore que partiellement connues. La production des DRO peut être déclenchée par des signaux de dommages tissulaires, comme une augmentation des concentrations en ions $\mathrm{Ca}^{2+}$ et d'ATP [33], ou la libération d'uracile par des microorganismes pathogènes qui s'introduisent dans l'organisme par la blessure qu'il a subi, comme cela a été mis en évidence chez la drosophile [34]. Des travaux menés chez cette espèce montrent que les DRO produits activent des macrophages produisant du TNF- $\alpha$ (tumor necrosis factor alpha) qui cible des cellules épithéliales proches de la blessure. Cela provoque l'entrée des cellules en apoptose et stimule la prolifération d'autres cellules via la voie de signalisation JNK (c-Jun N-terminal kinase) [29]. Chez le poisson-zèbre, les DRO induisent également une apoptose, et activent la voie JNK, stimulant la prolifération cellulaire nécessaire à la formation du blastème et l'activation d'autres voies de signalisation nécessaires à la morphogenèse de la structure qui va être ainsi régénérée [17].

${ }^{5}$ Xenopus laevis est une espèce d'amphibiens.

${ }^{6}$ L'axolotl, Ambystoma mexicanum, est une espèce de salamandre néoténique.

\section{Conclusions et perspectives}

Les différents travaux que nous avons présentés ont permis d'identifier un trio de mécanismes impliqués dans la régénération et nécessaires à sa réussite dans plusieurs espèces animales. La production de DRO, après une amputation, induit en effet le déclenchement d'un ou plusieurs épisode(s) d'apoptose qui, eux-mêmes, conduisent à une prolifération cellulaire compensatoire permettant de reformer les parties manquantes. Le fait de retrouver ce trio de mécanismes chez des espèces distantes les unes des autres d'un point de vue phylogénétique (par exemple la drosophile, l'hydre et le poisson-zèbre) et dans plusieurs types de régénération (régénération du corps entier, de structures complexes et d'organes), a conduit à formuler l'hypothèse de sa conservation au cours de l'évolution et suggère qu'il serait au cœur de l'initiation de la régénération chez tous les animaux ayant cette capacité.

Néanmoins, ces mécanismes sont mis en œuvre selon des modalités très variables d'un organisme à l'autre : la durée et la fréquence des phénomènes de mort et de prolifération cellulaire diffèrent en effet, de même que les voies de signalisation qui sont impliquées. Le rôle de ce trio (DRO-apoptose-prolifération) et les voies de signalisation qui participent à l'initiation de la régénération n'ont en fait pu être identifiés que dans un nombre très restreint d'espèces (Figure 3 ). Les données actuelles, encore parcellaires, nécessitent donc des études supplémentaires chez d'autres espèces d'animaux et s'intéressant à différents types de régénération. Il sera alors possible de déterminer si ce trio était déjà impliqué dans la régénération chez des ancêtres communs à de nombreux animaux éloignés en termes de phylogénie, ou s'il a été acquis indépendamment dans différentes espèces animales.

Chez l'homme, les capacités régénératives restent très limitées. L'étude des mécanismes de régénération présente donc un grand intérêt, en particulier pour le développement de la médecine régénérative. Ce domaine de recherche se développe selon deux axes principaux : la fabrication de greffons artificiels imitant les tissus à remplacer, et l'amélioration du potentiel régénératif intrinsèque chez l'homme. Cette seconde approche repose essentiellement sur des tentatives de reproduction du microenvironnement favorable à la régénération, comme celui retrouvé chez les animaux ayant une forte capacité de régénération [35]. Cela nécessite de caractériser ce microenvironnement dans différents modèles animaux. $\diamond$ 


\section{A}

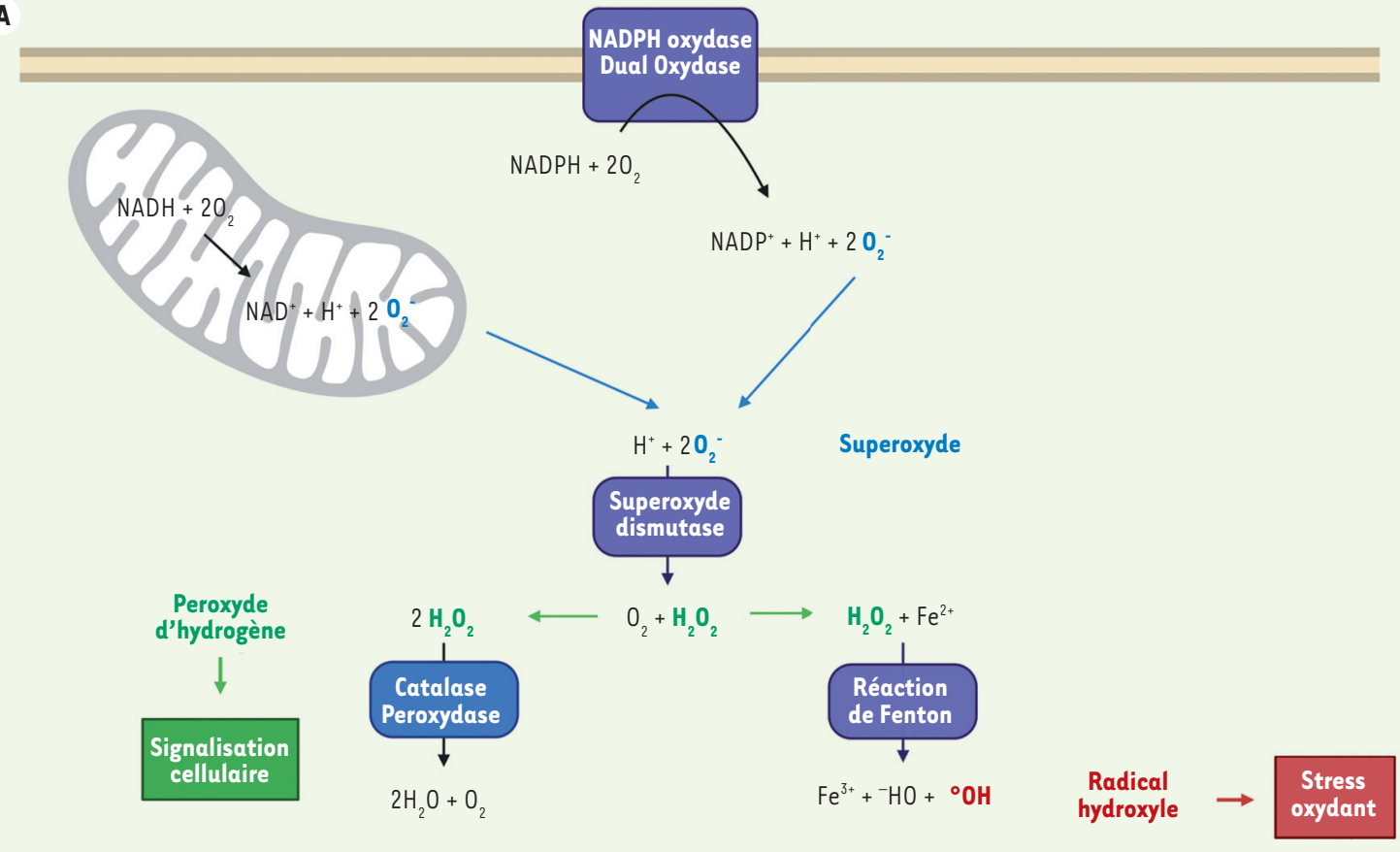

B1
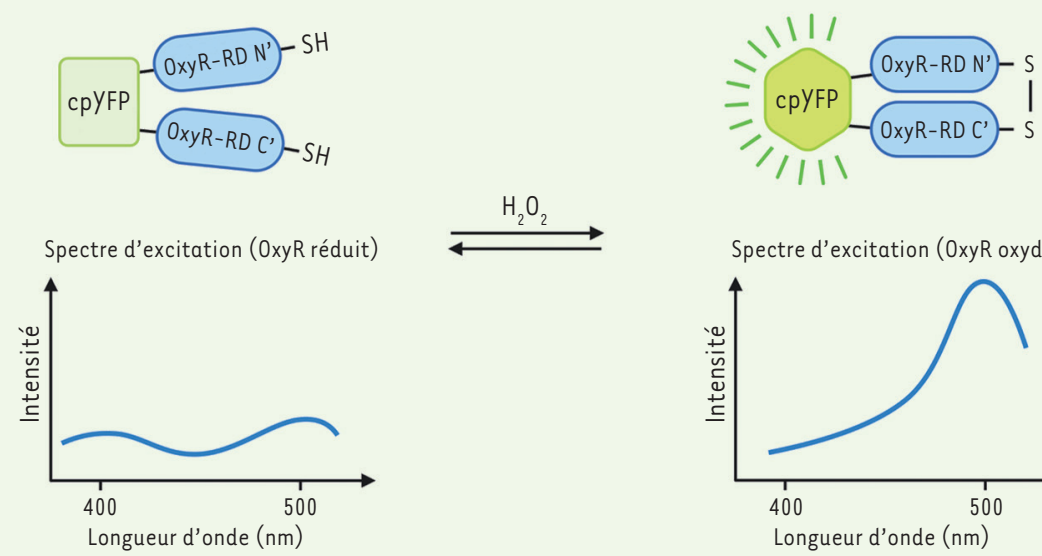

Spectre d'excitation (0xyR oxydé)

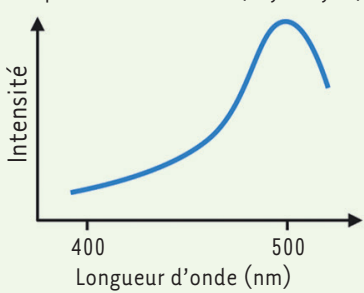

B2

Orpl $2_{S H}^{S H}{ }^{H S}-\overbrace{11}^{111 /}=$

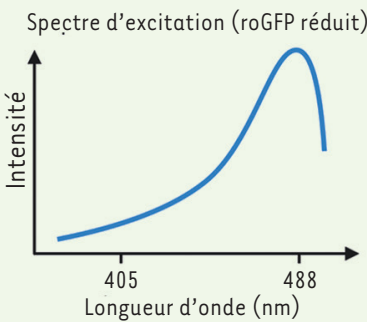

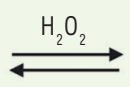
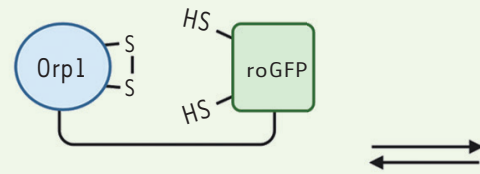

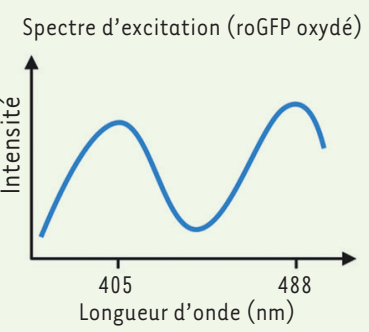


Figure 4. Principales voies du métabolisme des DRO et senseurs génétiques permettant leur étude. $A$. Stress oxydatif. Le superoxyde $\mathrm{O}_{2}{ }^{-}$est un produit secondaire de la chaîne respiratoire mitochondriale et de l'activité de certaines enzymes comme la NADPH oxydase (NOX) et la dual oxydase (Duox). L'enzyme appelée superoxyde dismutase ( $\mathrm{Sod}$ ) convertit $\mathrm{O}_{2}{ }^{-}$en $\mathrm{H}_{2} \mathrm{O}_{2}$ qui participe à diverses signalisations cellulaires et peut être converti en eau et dioxygène par des enzymes appelées catalases et peroxydases. $\mathrm{L}^{\prime} \mathrm{H}_{2} \mathrm{O}_{2}$ peut également donner naissance à des radicaux hydroxyles par la réaction de Fenton. Ces composés peuvent altérer tous types de macromolécules et causent des dommages cellulaires importants à l'origine du stress oxydant (schéma modifié d'après [39]). B. Les senseurs génétiques HyPer (B1) et roGFP (B2) permettent de mettre en évidence la présence et de quantifier I' $\mathrm{H}_{2} \mathrm{O}_{2}$ dans les tissus vivants. (B1) Le senseur HyPer a été mis au point dans des cultures cellulaires humaines et murines par Belousov et al. en 2006 [30] et a été utilisé in vitro et in vivo chez plusieurs animaux, notamment le xénope [28], la souris [40] et le poisson-zèbre [41]. Il est constitué d'une protéine, appelée cpYFP (circularly permuted yellow fluorescent protein), insérée dans le domaine régulateur (RD) de la protéine procaryote $0 x y R$ (un facteur de transcription activé par l' $\mathrm{H}_{2} \mathrm{O}_{2}$ ). L'ensemble est peu excitable en l'absence d' $\mathrm{H}_{2} \mathrm{O}_{2}$. En revanche, en présence d' $\mathrm{H}_{2} \mathrm{O}_{2}$, deux cystéines d'Oxy- $\mathrm{RD}$ vont former un pont disulfure intramoléculaire (S-S), modifiant ainsi la conformation de la cPYFP et son spectre d'excitation (pic à $500 \mathrm{~nm}$ ). Le suivi de la fluorescence émise permet ainsi de détecter la présence d' $\mathrm{H}_{2} \mathrm{O}_{2}$. (B2) Le senseur roGFP est constitué de deux protéines, Orpl et roGFP [42]. Orpl est très réactive à l' $\mathrm{H}_{2} \mathrm{O}_{2}$. En sa présence, elle est d'abord oxydée (induisant la formation d'un pont disulfure $\mathrm{S}-\mathrm{S}$ ) puis joue le rôle de relai oxydant, en oxydant des protéines cibles proches, ici roGFP. roGFP a alors un spectre d'excitation changé: alors qu'à l'état réduit, il n'a qu'un pic d'excitation à $488 \mathrm{~nm}$, après oxydation, il présente deux pics d'intensité équivalents à 405 et $488 \mathrm{~nm}$. L'évolution de la quantité de fluorescence émise à chacune de ces longueurs d'onde permet d'établir la présence d' $\mathrm{H}_{2} \mathrm{O}_{2}$ dans les cellules.

\section{SUMMARY}

A trio of mechanisms involved in regeneration initiation in animals Whole-body and complex structure regeneration is a widespread phenomenon in animals. While regenerative abilities vary greatly from one species to another, a number of mechanisms appear essential for regeneration in distantly related phylogenetic groups. In this review, we synthetize the knowledge gathered on the implication of three mechanisms that appear to be important for the initiation of regeneration in animals. Reactive Oxygen Species (ROS) are metabolic by-products involved in cell signalling, which are produced shortly after amputation in several species. ROS production may be responsible for triggering apoptosis, another recurring mechanism involved in regeneration initiation. In turn, apoptosis causes compensatory proliferation by setting off cellular division, thus contributing to the reconstitution of tissues. Inhibiting either ROS production, apoptosis or cellular proliferation impairs regeneration in a variety of model species. $\diamond$

\section{FINANCEMENTS \&T SOUTIENS}

Nos travaux sont financés par le CNRS, l'Université de Paris, l'Université Côte d'Azur, l'agence nationale de la recherche (ANR), la ligue nationale contre le cancer (LNCC), l'association pour la recherche sur le cancer (ARC), La Fondation pour la recherche médi-

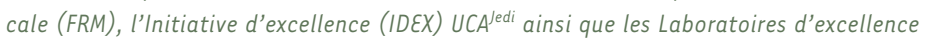
(LABEX) «Who Am I ? » et «SIGNALIFE » qui bénéficient d'un financement de l'État géré par l'ANR, dans le cadre des investissements d'avenir.

\section{LIENS D'INTÉRÊT}

Les auteurs déclarent n'avoir aucun lien d'intérêt concernant les données publiées dans cet article.

\section{RéFÉRENCES}

1. Poss KD. Advances in understanding tissue regenerative capacity and mechanisms in animals. Nat Rev Genet $2010 ; 11: 710-22$.

2. Vervoort M. Regeneration and development in animals. Biological Theory $2011 ; 6: 25-35$.

3. Chen CH, Poss KD. Regeneration genetics. Annu Rev Genet 2017 ; $51: 63-82$.

4. Tiozzo $S$, Copley RR. Reconsidering regeneration in metazoans: an evo-devo approach. Front $\varepsilon$ col Evol 2015 ; 3. doi: 10.3389/fevo.2015.00067.

5. Amiel AR, Johnston HT, Nedoncelle $K$, et al. Characterization of morphological and cellular events underlying oral regeneration in the sea anemone, Nematostella vectensis. Int J Mol Sci 2015 ; 16 : 28449-71.

6. Planques A, Malem J, Parapar J, et al. Morphological, cellular and molecular characterization of posterior regeneration in the marine annelid Platynereis dumerilii. Dev Biol $2019 ; 445: 189-210$.

7. Ramon-Mateu J, Ellison ST, Angelini TE, Martindale MQ. Regeneration in the ctenophore Mnemiopsis leidyi occurs in the absence of a blastema, requires cell division, and is temporally separable from wound healing. BMC Biol $2019 ; 17: 80$.

8. Amiel AR, Foucher K, Ferreira S, Röttinger $\varepsilon$. Synergic coordination of stem cells is required to induce a regenerative response in anthozoan cnidarians. BioRxiv 2019. doi. https://doi: org/10.1101/2019.12.31.891804

9. Mcllwain DR, Berger T, Mak TW. Caspase functions in cell death and disease. Cold Spring Harb Perspect Biol 2013 ; 5 : a008656.

10. Saunders JW, Fallon JF. Cell death in morphogenesis. In : Locke M, ed. Major problems in developmental biology. New Yok : Academic Press, 1966 : 289-314.

11. Vriz S, Reiter S, Galliot B. Cell death: a program to regenerate. Curr Top Dev Biol 2014 ; 108 : 121-51.

12. Krasovec G, Robine K, Queinnec $\varepsilon$, et al. Ci-hoxl2 tail gradient precedes and participates in the control of the apoptotic-dependent tail regression during Ciona larva metamorphosis. Dev Biol 2019 ; 448 : 237-46.

13. Bardet PL, Kolahgar G, Mynett A, et al. A fluorescent reporter of caspase activity for live imaging. Proc Natl Acad Sci USA 2008 ; 105 : 13901-5.

14. Ding Y, Li J, Enterina JR, et al. Ratiometric biosensors based on dimerizationdependent fluorescent protein exchange. Nat Methods 2015 ; 12 : 195-8.

15. Chera S, Ghila L, Dobretz K, et al. Apoptotic cells provide an unexpected source of Wnt3 signaling to drive hydra head regeneration. Dev Cell 2009 ; $17: 279-89$.

16. Warner JF, Amiel A, Johnston H, Rottinger $\varepsilon$. Regeneration is a partial redeployment of the embryonic gene network. BioRxiv 2019. doi: https://doi.org/10.1101/658930

17. Gauron C, Rampon C, Bouzaffour M, et al. Sustained production of ROS triggers compensatory proliferation and is required for regeneration to proceed. Sci Rep 2013 ; 3 : 2084.

18. Milan M, Campuzano S, Garcia-Bellido A. Developmental parameters of cell death in the wing disc of Drosophila. Proc Natl Acad Sci USA 1997 ; 94 : 5691-6.

19. Perez-Garijo A, Steller H. Spreading the word: non-autonomous effects of apoptosis during development, regeneration and disease. Development $2015 ; 142: 3253-62$

20. Fan Y, Bergmann A. Apoptosis-induced compensatory proliferation. The cell is dead. Iong live the cell! Trends Cell Biol $2008 ; 18: 467-73$.

21. Godwin JW, Pinto AR, Rosenthal NA. Macrophages are required for adult salamander limb regeneration. Proc Natl Acad Sci USA 2013; 110 : 9415-20.

22. Ricci L, Srivastava M. Wound-induced cell proliferation during animal regeneration. Wiley Interdiscip Rev Dev Biol 2018 : e321.

23. Wiese KE, Nusse R, van Amerongen R. Wnt signalling: conquering complexity. Development $2018 ; 145$ : dev165902.

24. Brock CK, Wallin ST, Ruiz $0 E$, et al. Stem cell proliferation is induced by apoptotic bodies from dying cells during epithelial tissue maintenance. Nat Commun $2019 ; 10: 1044$ 


\section{RÉFÉRENCES}

25. Medina CB, Mehrotra P, Arandjelovic $S$, et al. Metabolites released from apoptotic cells act as tissue messengers. Nature $2020 ; 580: 130-5$.

26. Rampon C, Gauron C, Meda F, et al. Adenosine enhances progenitor cell recruitment and nerve growth via its A2B receptor during adult fin regeneration. Purinergic Signal $2014 ; 10: 595-602$.

27. Reczek CR, Chandel NS. ROS-dependent signal transduction. Curr Opin Cell Biol $2015 ; 33: 8-13$.

28. Love NR, Chen $Y$, Ishibashi $S$, et al. Amputation-induced reactive oxygen species are required for successful Xenopus tadpole tail regeneration. Nat Cell Biol 2013; $15: 222-8$.

29. Fogarty CE, Diwanji N, Lindblad JL, et al. Extracellular reactive oxygen species drive apoptosisinduced proliferation via Drosophila macrophages. Curr Biol $2016 ; 26$ : 575-84.

30. Belousov VV, Fradkov AF, Lukyanov KA, et al. Genetically encoded fluorescent indicator for intracellular hydrogen peroxide. Nat Methods $2006 ; 3: 281-6$.

31. Hanson GT, Aggeler R, Oglesbee D, et al. Investigating mitochondrial redox potential with redoxsensitive green fluorescent protein indicators. J Biol Chem $2004 ; 279$ : 13044-53.

32. Al Haj Baddar NW, Chithrala A, Voss SR. Amputation-induced reactive oxygen species signaling is required for axolotl tail regeneration. Dev Dyn $2019 ; 248$ : 189-96.

33. Cordeiro JV, Jacinto A. The role of transcription-independent damage signals in the initiation of epithelial wound healing. Nat Rev Mol Cell Biol $2013 ; 14$ : 249-62.

34. Buchon N, Broderick NA, Lemaitre B. Gut homeostasis in a microbial world: insights from Drosophila melanogaster. Nat Rev Microbiol 2013 ; 11 : 615-26.

35. Mao AS, Mooney DJ. Regenerative medicine: current therapies and future directions. Proc Natl Acad Sci USA 2015 ; 112 : 14452-9.
36. Bely AE, Nyberg KG. Evolution of animal regeneration: re-emergence of a field. Trends Ecol Evol $2010 ; 25$ : 161-70.

37. Bergmann A, Steller H. Apoptosis, stem cells, and tissue regeneration. Sci Signal $2010 ; 3$ : re8.

38. Galluzzi L, Vitale I, Abrams JM, et al. Molecular definitions of cell death subroutines: recommendations of the nomenclature committee on cell death 2012. Cell Death Differ $2012 ; 19$ : 107-20.

39. Coffman JA, Su YH. Redox regulation of development and regeneration. Curr Opin Genet Dev 2019; $57: 9-15$

40. Staunton CA, Owen ED, Pollock N, et al. HyPer2 imaging reveals temporal and heterogeneous hydrogen peroxide changes in denervated and aged skeletal muscle fibers in vivo. Sci Rep 2019; 9 : 14461.

41. Zou Y, Wang A, Shi M, et al. Analysis of redox landscapes and dynamics in living cells and in vivo using genetically encoded fluorescent sensors. Nat Protoc $2018 ; 13: 2362-86$.

42. Gutscher M, Sobotta MC, Wabnitz GH, et al. Proximity-based protein thiol oxidation by H202-scavenging peroxidases. J Biol Chem 2009 ; 284 : 31532-40.

43. Vieillissement et mort : de la cellule à l'individu. Med Sci (Paris) 2020 ; 36 : 1103-238.

\section{TIRÉS À PART}

દ. Gazave

B ertrand Jordan a participé à l'extraordinaire aventure de la biologie moléculaire, encore balbutiante dans les années 1960, mais qui a complètement révolutionné les sciences du vivant au cours des décennies suivantes.

L'ADN, quasiment inaccessible au début de cette période, intervient maintenant dans d'innombrables recherches, de l'écologie à l'anthropologie, sans oublier bien sûr la médecine dont il fait désormais partie intégrante. Nous pouvons aujourd'hui lire intégralement
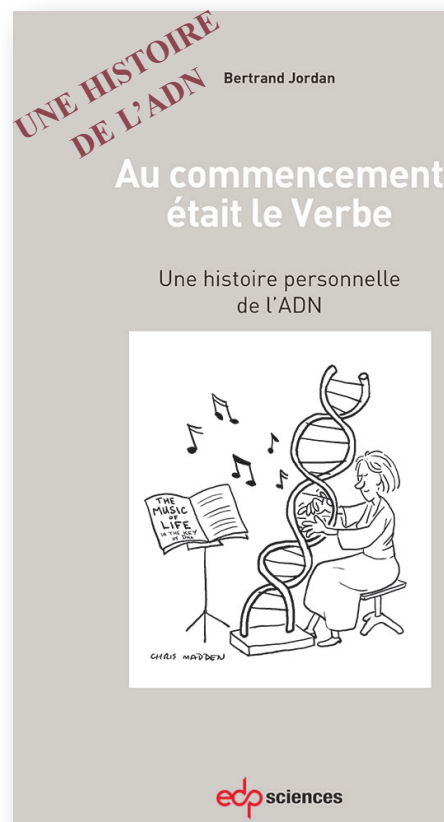

SBN : 978-2-7598-1710-8
I'ADN d'une personne en quelques heures, et en tirer de précieuses informations pour la prévention et le traitement de nombreuses maladies - et nous n'en sommes qu'au début de cette nouvelle médecine !

Acteur mais aussi témoin de ces avancées, Bertrand Jordan fait ici un récit très personnel et sans langue de bois de sa vie avec l'ADN. Ce livre ne prétend pas être une histoire complète de la biologie moléculaire, mais il illustre son développement, révélant parfois le «dessous des cartes» grâce aux expériences vécues par son auteur.

\section{Retrouvez toutes les Actualités de la Myologie sur les sites de :}

la Société Française de Myologie

www.sfmyologie.org
À retourner à EDP Sciences, 17 avenue Hoggar, 91944 Les Ulis

Tél. : 0149856069 - Fax : 0149850345 - $\varepsilon$-mail : francois.flori@edpsciences.org

NOM : .......................................................... Prénom

Adresse

Code postal : ................................................. Ville :

Pays :

Fonction :

Je souhaite recevoir

Au commencement était le Verbe : $20 €+3 €$ de port $=23 € \pi \mathrm{C}$

en ................. exemplaire, soit un total de

$\square$ Par carte bancaire : $\quad \square$ Visa $\square$ Eurocard/Mastercard

Carte $\mathrm{n}^{\circ}$ ! I ! I I ! I I I ! I I I ! ! I I I I

Date d'expiration : ! I ! I I $\mathrm{N}^{\circ}$ de contrôle au dos de la carte : ! I I ! I I Signature: la filière de santé neuromusculaire FILNEMUS

www. filnemus.fr 